\title{
CAD-BASED PATH PLANNING FOR LINE LASER SCANNING OF CURVED SURFACE
}

\author{
W. Guo ${ }^{1 *}$, Z. Zhao ${ }^{1}$, D. Ding ${ }^{1}$, Y. Fu ${ }^{1}$ \\ ${ }^{1}$ Nanjing University of Aeronautics and Astronautics, College of Mechanical and Electrical Engineering, Nanjing, \\ China \\ *Corresponding author; e-mail: zhengcaizhao@nuaa.edu.cn
}

\begin{abstract}
On-machine measurement by a line laser scanner has a great potential in machining large complex components in aerospace and automation applications. This paper proposes a novel approach for path planning of measuring curved surfaces in a 5-axis machine tool, which takes scanning overlap, visual angle, and working distance into consideration. A conformal mapping algorithm is employed to transform a three-dimensional (3D) mesh surface to a 2D parametric plane. The shortest equidistant paths calculated in the 2D plane are transformed into smooth collision-free scanning paths in the 3D surface. The experiments verify that this method can improve the efficiency by $21.4 \%$ and the accuracy by $24.9 \%$. Keywords:

Laser-based on-machine measurement; Scan path planning; Conformal mapping; 5-axis machine tool
\end{abstract}

\section{INTRODUCTION}

With the development of digitalization in modern industry, the mechanical parts with complex curved surfaces have higher precision in mechanics, optics, and fluid science. But the uniformity of blank forming and the uneven distribution of machining allowance results in a high reject rate of parts with complex surfaces [1]. Therefore, it is necessary to obtain accurate actual blank shape measurement data to guide NC Machining to correct the shape of the blank. A three-coordinate measuring machine (CMM) has the advantages of high measuring accuracy, reliable performance, and large off-line measuring range, the repeated clamping and position movement of parts have an important influence on the precision and efficiency of measurement [2]. Therefore, on-machine measurement (OMM) is proposed to obtain the geometric information of complex parts without removing the workpiece from the machine tool system [3]. As part of the Industry 4.0 measurement technology, OMM can efficiently compare the geometric consistency of the workpiece and adjust the process [4]. At present, there are two methods based on probe and laser. Laser OMM has become the preferred solution due to its stable accuracy, high efficiency, without contact, and other characteristics [5]. The integration of laser OMM technology and machining has become an important process for high quality and high-efficiency machining of complex surface parts. But so far, little research has been done on on-machine scanning path planning with integrated linear laser sensors. This paper proposes an approach for path planning of measuring curved surfaces using a line laser scanner for measurement on a 5-axis machine tool.

Recently, many researchers have examined the scanning path planning based on the CAD model to obtain the completed surface point clouds with low noise efficiently. From the CAD model, Mahmud et al. [6] proposed an approach based on the concept of visibility to adjust the angle between the laser plane and the scanned surface. Son et al. [7] considered various scanning parameters in the generation of optimal scan paths, such as the view angle, depth of field, field of view (FOV), and occlusion. Xi and Shu et al. [8] divided a part CAD model into several sections and determined the optimal parameters of the scanner FOV to ensure maximum coverage for each scanned section. These researches are based on CMM to realize the measurement of the curved surface by the linear laser scanner. Wu et al. [9] propose a CAD model of a triangular mesh path planning method for surface inspection on a robot-based scanning system. Some constraints of the structured light scanner are taken into account, i.e. FOV, visual angle, working distance, and overlap. The first step of this case is to extract the model wireframe from the CAD model in the main direction by computing the mean of all the normal vectors of the triangular mesh. Secondly, the model is divided into several regions based on the rectangle framework where viewpoints satisfied scan constraints are generated. But the scan region segmentation should be more consistent with the surface characteristics, it should be a small section of the irregular surface but rectangular. Phan et al. [10] control the overlap of scan paths to ensure the measurement quality with a laser-plane scanner mounted on an industrial robot. In this case, the continuous scan path was built from a set of viewpoints, which allows the management of both the orientation and the coverage rate of the laser beam. But there is a sudden change of scanner orientation when the scanner moves from one point to another. This will result in scanning part of the redundant area and missing part of the surface information. These studies are based on a 6-degree 
freedom industrial robot to achieve the curved surface of the line laser scanner measurement. Therefore, the use of an optimal scanning strategy with a line laser sensor is still a challenge especially adapted to 5 -axis machine tools.

The measurement time and accuracy are core issues for OMM that exist in the process between machining operations, which reflects the necessity of measurement path planning. There are three main factors that affect the scanning accuracy of the sensor, including sensor parameters, scanning process, and part surface. Specifically, the sensor parameters are mainly about measurement accuracy. The surface parameters of the part are related to the material and surface texture. The scanning process parameters play an essential role which refers to the scan path and motion accuracy.

According to the analysis of the CAD surface model and the sensor scanning parameters, this paper proposes a strategy to plan a smooth scan path for complex curved surfaces to improve measurement accuracy and efficiency.

\section{SCAN PATH PLANNING}

The scanning path is composed of measuring planning points, measuring path points, and scanner orientations, in which each measuring path point corresponds to a certain scanner orientation. The scanning path is the integration of a series of measurement path points and scanner orientation. In addition, in order to obtain the completed surface information considering the quality and efficiency, it is necessary to optimize the scanning overlap, the path length, and the scanner orientation. The overall procedure for the generation of scan path gives as follows:

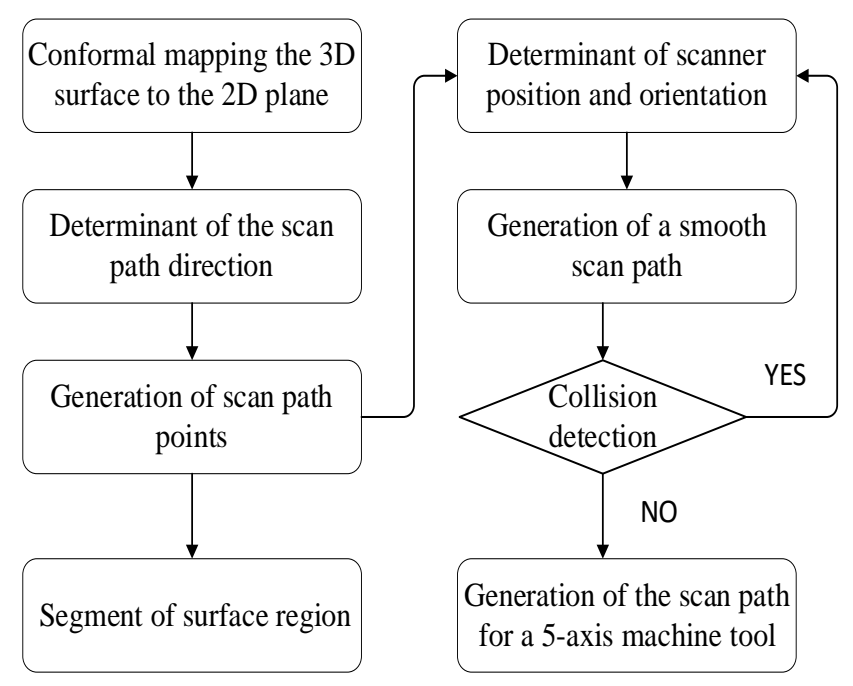

Fig. 1: Overall procedure for the generation of scan path.

According to the light beam characteristics of the line laser scanner, the scanning path is defined by the following parameters. The vector VL and VN determine the scanning orientation of the incident laser beam, in which VL is the direction vector of the laser scanning line and VN is the direction vector of the beam axis. The laser line length $L$ determines the laser line scanning range, while the line laser scanning height $\mathrm{H}$ and the working distance range $\mathrm{Hf}$ determine the FOV (Fig. 2).

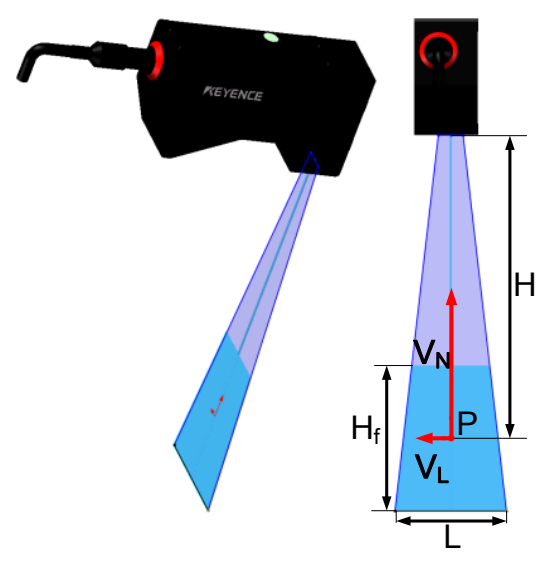

Fig. 2: 3D line laser scanning parameterization.

For the curved surface scanning problem, planning the scanning path is essential to obtain a set of continuous measurement planning points $(P)$ and the corresponding orientation $\left(\mathbf{V}_{\mathbf{N}}, \mathbf{V}_{\mathbf{L}}\right)$ of the scanner at each planning point. Defining the scan path in this way, we address the problem of path planning for the 3D line laser scanning from the point of view of inspection, which is similar to generate the toolpath for computer numerical control (CNC)based on CAD model. the iso-scallop tool path method is proposed to predict and adjust the distance between adjacent tool paths to avoid the undercut and excessive overlap [11]. The method can predict the following path of the driveline according to the existing tool path, then construct the effective overlapping interval and select several characteristic tool positions to represent the whole path. Finally, the iso-scallop is achieved between the adjacent tool paths. The difference lies in that the toolpath is to drive a point along a given curve, while the scanning path is to drive a line across a given surface to sweep through a surface with a maximum coverage [8]. Inspired by the isoscallop tool path method used for machining, the isooverlap scan path method focus on the conformal map is applied to control the scanning overlap of two adjacent paths (Fig. 3).

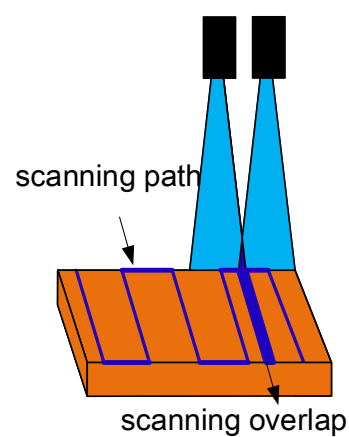

Fig. 3: Definition of the scanning overlap.

Furthermore, in order to plan the measured trajectory in the parameter domain, the surface of the object must be flattened to 2D space in order to simplify the calculation. The triangular mesh surface is similar to the original surface and has good network topology property. Especially for the surfaces such as mesh surface and point cloud surface, it is necessary to use the conformal mapping method to transform the surface into a mesh plane, so as to carry on the research of subsequent scanning trajectory [12]. 


\subsection{Iso-overlap scan path method using conformal mapping}

First, conformal mapping is an optimization constrained only by the angle. The position of the vertices of the plane mesh is calculated by solving the minimum value of the angle change of the plane mesh relative to the origina surface. Some parameters are set in table 1 to define the function:

Tab. 1: 2D mesh and 3D mesh parameters.

\begin{tabular}{ll}
\hline 2D mesh parameters & 3D mesh parameters \\
\hline $\mathrm{S}_{\mathrm{f}}$, set of $\mathrm{n}$ facets & $\mathrm{S}_{\mathrm{f}}$, set of $\mathrm{n}$ facets \\
$\mathrm{f}_{i}$, facet $\mathrm{I}, \mathrm{f}_{i} \in \mathrm{S}_{\mathrm{f}}, \mathrm{i} \in[1, \mathrm{n}]$ & $\mathrm{F}_{i}$, facet $\mathrm{i}, \mathrm{F}_{i} \in \mathrm{S}_{\mathrm{f}}, i \in[1, \mathrm{n}]$ \\
$\mathrm{S}_{\mathrm{v}}$, set of $\mathrm{m}$ vertices & $\mathrm{S}_{\mathrm{v}}$, set of $\mathrm{m}$ vertices \\
$\mathrm{v}_{k}$, vertex of the mesh, $\mathrm{v}_{k} \in$ & $\mathrm{V}_{k}$, vertex of the mesh, $\mathrm{V}_{k}$ \\
$\mathrm{~S}_{\mathrm{v}}, k \in[1, \mathrm{~m}]$ & $\in \mathrm{S}_{\mathrm{v}}, k \in[1, \mathrm{~m}]$ \\
$\mathrm{af}_{i}$, area of facet $\mathrm{f}_{i}$ & $\mathrm{AF}_{i}$, area of facet $\mathrm{F}_{i}$ \\
$\alpha_{i}^{\mathrm{j}(k)}$, angle of the facet $\mathrm{f}_{i}, i$ & $\beta_{i}^{\mathrm{j}(\mathrm{k})}$, angle of the facet $\mathrm{F}_{i}$, \\
$\in[1, \mathrm{n}], j=1$ to3, $k \in[1, \mathrm{~m}]$ & $i \in[1, \mathrm{n}], j=1$ to3, $k \in[1, \mathrm{~m}]$
\end{tabular}

The basic mathematical formula of the conformal mapping algorithm calculated as follows:

$E(\alpha)=\sum_{i=1}^{n} \sum_{j=1}^{3}\left(\alpha_{i}^{j}-\phi_{i}^{j}\right)^{2} w_{i}^{j}$

Where denote the relative change in angle and its initial value is $\left(\phi_{i}^{j}\right)^{-2}$. $\phi_{i}^{j}$ denote optimized angle value which correspond to and its value depends on where the vertex is. This gives:

$$
\phi_{i}^{j}(k)= \begin{cases}\beta_{i}^{j(k)} \frac{2 \pi}{\sum_{i} \beta_{i}^{j(k)}} & \text { when } \mathrm{V}_{\mathrm{k}} \text { is interior point } \\ \beta_{i}^{j(k)} & \text { when } \mathrm{V}_{\mathrm{k}} \text { is boundary point }\end{cases}
$$

Where $\sum_{i} \beta_{i}^{j(k)}$ denote the sum of the angles in a surface mesh with $\mathrm{V}_{K}$ as the vertex.

In order to ensure the validity of the parameterized angle, several constraints must be met in solving the objective function. The first condition (see equation (3)) is that the sum of the interior angles of the triangles mapped to the plane is $\pi$, which determines the validity of the triangle. The second condition (see equation (4)) is that the interior vertices are of the same planarity, while the third condition (see equation (5)) is that the common edges of adjacent triangular meshes are of the same length. These conditions ensure the validity of the topological complementarity relation of planar grids. These conditions can be expressed by:

$g_{i}^{(1)} \equiv \alpha_{i}^{1}+\alpha_{i}^{2}+\alpha_{i}^{3}-\pi=0, i=1 \ldots n$

$g_{k}^{(2)} \equiv \sum_{i} \alpha_{i}^{j(k)}-2 \pi=0, k=1 \ldots m$

$g_{k}^{(3)} \equiv \frac{\prod_{i} \sin \left(\alpha_{i}^{j(k)+1}\right)}{\prod_{i} \sin \left(\alpha_{i}^{j(k)-1}\right)}-1=0, k=1 . . . m$

if $j(k)=3$, then $j(k)+1=1$; if $j(k)=1$, then $j(k)-1=3$.

We use Newton's method to solve the optimal solution of the objective function which minimize. After the implementation of the conformal mapping algorithm, the set $\mathrm{Sf}$ of $\mathrm{n}$ triangular facets is transformed into the set sf of $n$ triangular facets. Then a 3D mesh surface is stretched on a
2D parametric plane for simplifying the calculation of the scanner trajectory (Fig. 5). The next step is to plan the measurement points in the $2 \mathrm{D}$ plane according to the scanning strategy and generate equidistant and overlapping scanning paths. Eventually, the iso-overlap scan paths are obtained by inverse mapping to the 3D surface.

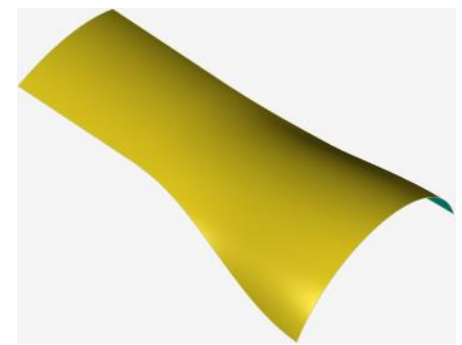

Fig. 4: A CAD model of the 3D complex curved surface.

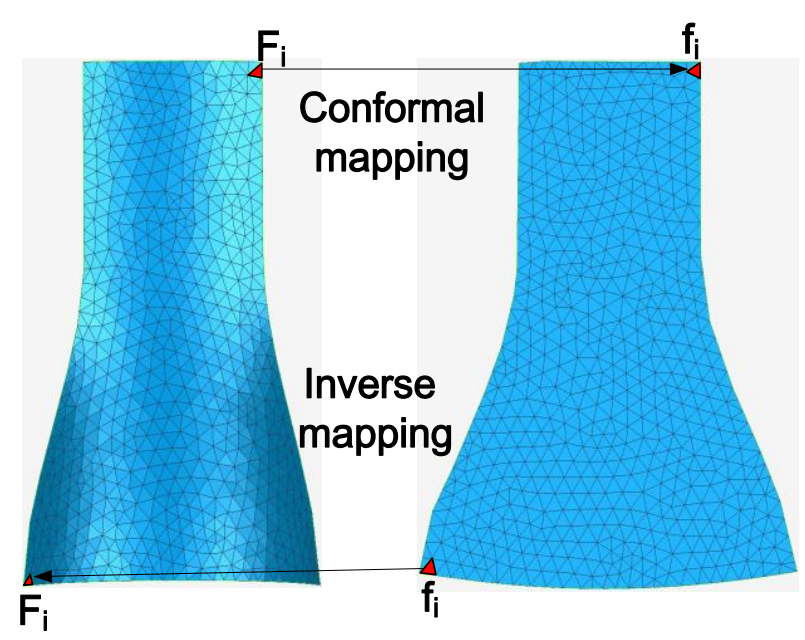

Fig. 5: Transformation between 3D surface and 2D plane.

\subsection{Measuring planning point generation using oriented bounding box algorithm}

There is a strict requirement for measuring efficiency in the actual OMM process. The key to scanning path planning is to complete the measurement of the surface with a shorter total path and as few adjustments as possible. In order to minimize the measuring time, the laser line must be perpendicular to the direction of displacement along the scanner trajectory in the 3D space [10]. In essence, the measuring displacement direction of the laser sensor path must be the main direction of the surface, so as to ensure that the scanning path is the shortest. We use PCA (Principal Component Analysis) method to reduce the dimension of $2 \mathrm{D}$ surface mesh vertices data $(\mathrm{x}, \mathrm{y})$ by a linear transformation, and extract the main feature component, which is the main direction of the surface.

In 2D Space, the bounding box of the surface is established according to the principal direction of the surface. The measuring point is the intersection point of the measuring path and the edge of the triangle surface, which is formed by the line width and the overlapped lines of the line laser. Conformal mapping only ensures that the shape of the surface remains the same and that the dimensions change proportionally. Therefore, the line length I of the line laser in $2 \mathrm{D}$ space is proportional to the line length $L$ of the line laser in $3 \mathrm{D}$ space. This proportional can be expressed by: 


$$
\frac{L}{l}=\sqrt{\frac{1}{n} \sum_{i=1}^{n} \frac{A_{F i}}{a_{f i}}}
$$

After the line laser length in 2D space is obtained by equation (6), the shortest equidistant path can be planned in the oriented bounding box when the overlapping requirement of scanning is given. Then the intersection points of the surface triangular mesh and equidistant path in the parameter space are $p_{i}\left(p_{1}, p_{2} \ldots p_{n}\right)$. Since conformal mapping is a reversible process, the $2 \mathrm{D}$ points pi is transformed into measurement planning point $P_{i}\left(P_{1}\right.$, $\mathrm{P}_{2} \ldots \mathrm{P}_{n}$ ) of $3 d$ space surface by inverse mapping.

When the coordinates of all measurement planning points are known, the corresponding scanner position for each path point should also be determined. The directors $V_{\mathbf{L}}$ and $\mathbf{V}_{\mathbf{N}}$ of the scanner determine the position of the scanner. The next section first determines the direction vector $\mathbf{V}_{\mathbf{L}}$ of the scanning line and then the direction vector $\mathbf{V}_{\mathbf{N}}$ of the light beam axis.

\subsection{The determinant of scanner position using the region growing method}

As mentioned above, the direction of the laser scan line should be perpendicular to the displacement along the scanner trajectory in the $3 d$ space. Meanwhile, the direction of the scan line in 2D space must be perpendicular to the path. Then laser line is positioned perpendicularly to the trajectory and centered at pi. By inverse mapping the starting point $p_{i s}$ and the ending point $p_{i e}$ of the laser scanning line in $2 D$ space into $3 D$ space, $V_{\mathbf{L}}$ can be calculated from $P_{\text {is }}$ and Pie of the laser scanning line in $3 D$ space.

In order to ensure the high precision of laser measurement, the beam axis should be perpendicular to the surface as far as possible. However, because of the structure of the laser line, it is impossible to make the line perpendicular to the surface everywhere in one scanner orientation. Therefore, we calculate the mean normal of the surface triangular facets corresponding to the laser scanning line at $P_{i}$, which represents the normal vector $\mathbf{V}_{\mathbf{N}}$ of the laser scanning line (Fig.6.).

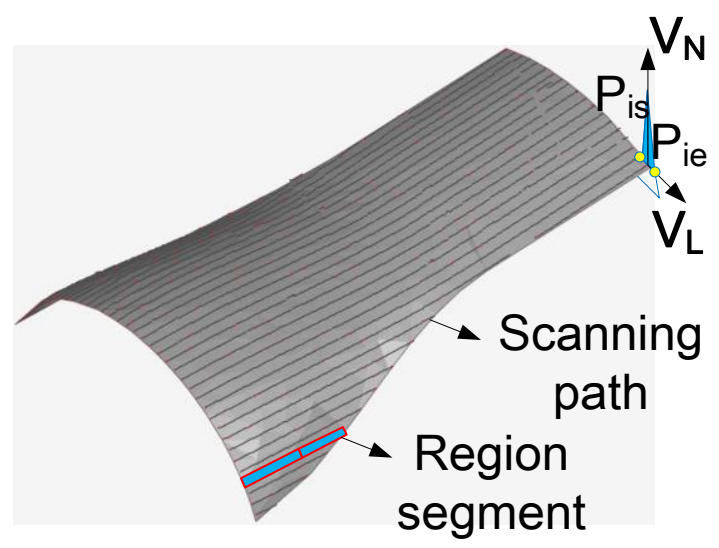

Fig. 6: Scanner position with the region segment.

In the real OMM process, the measurement is effective when the viewpoints are located in the FOV. In order to avoid adjusting the position of the scanner many times, we should select the characteristic measurement point to complete the scanning measurement of a region. Through the analysis of surface curvature and normal vector, the region growing method is used to filter feature measurement points that meet the visual angle and working distance constraints of the sensor to adjust the scanning position. The region growing method is divided into three steps. The first step is to identify the growing seed, which is the starting point of the current area scan path. The second step is to determine the region growth process including other similar growth criteria. In this case, the angle between the normal vector of the surface point and the incident light should be less than the threshold value. The third step is to determine the termination conditions in the growth process. In laser measurement, the angle between the normal vector of the endpoint and the normal vector of the starting point of the region should be less than the limit angle specified by the scanner. Besides, the measured data will be lost when the working distance is out of range. After the implementation of the region growing algorithm, the measuring path generates $\mathrm{k}$ regions, which means the laser scanner completes the scanning measurement under $k$ positions. The measuring point set under each measuring position is $\mathrm{P}_{j}, \mathrm{P}_{j} \in \mathrm{P}_{i}$.

\subsection{Generation of smooth collision-free scanning path for a 5-axis machine tool}

Although in this method, the free-form surface can be scanned in the shortest time, when the scanner moves from one point to another, the orientation of the scanner will suddenly change from the normal direction of the previous point to the current point, which may result in excessive scanning or missing some regions. This issue deal with the smooth continuous scanning path obtained by the cubic Bspline interpolation method. In the context of scanning path planning, collision and beam occlusion detection must be completed, so as to ensure the safety and effectiveness of the measurement process. Taking into account the reflected light as well as sensor geometry, an oriented bounding box algorithm is employed to detect the collision with the workpiece. Furthermore, the smooth collision-free scanning path $\left(\mathrm{P}, \mathbf{V}_{\mathbf{N}}, \mathbf{V}_{\mathbf{L}}\right)$ is a series of position points and beam direction in the workpiece frame. Where $\mathbf{V}_{\mathbf{L}}$ is perpendicular to the main direction of the oriented bounding box of the curve surface. The scanning path points are expressed as $\left(X_{p}, Y_{p}, Z_{p}, N_{i}, N_{j}, N_{k}\right)$. In order to realize the optimal scanning strategy of OMM, the 6-degree freedom coordinate must be converted to a 5-axis coordinate system $\left(X_{m}, Y_{m}, Z_{m}, B, C\right)$. In this section, the smooth method and measurement validity test are presented first. Eventually, the coordinate transformation is interpreted to apply to a 5axis machine tool.

The cubic B spline curve has good controllability and smoothness, and its basic function is simple to calculate. Therefore, the path curve can be fitted by 3-degree B-spline curve interpolation from the planned path point $\mathrm{Pi}$. In the last section, the feature measurement points were filtered by region segment. B-spline interpolation is used to smooth the path between two feature measurement points. It is necessary to explain the calculation process of cubic bspline interpolation. Firstly, the start and end data points are taken as the first and last points of the curve, and the node points correspond to the data points one by one. Then, the b-spline basis function is obtained by Deboor recursive algorithm. Finally, the control points of the path curve are obtained through the B-spline curve formula and the planned path point $\mathrm{P}_{\mathrm{i}}$. According to the above calculation we get the scanning path cubic $b$ spline curve formula as equation (7):

$$
P(u)=\sum_{i=0}^{n} d_{i} N_{i, k}(u), u \in\left[u_{k}, u_{n+1}\right]
$$

Where $d_{i}\left(d_{1}, d_{2} \ldots d_{n}\right)$ denotes the control points, and $N_{i, k}(u)$ is the $\mathrm{k}$ degree $\mathrm{b}$-spline basis function which is k-degree polynomial determined by node vector $U\left(u_{0}<u_{1}<\ldots<u_{n}\right)$. The $\mathrm{P}(\mathrm{u})$ is calculated according to the B-spline curve formula, 
and the smooth path is generated by interpolation between two feature points.

The OBB method is used to test the effectiveness of the smoothed path. For collision detection, the geometry of the scanner and the measured workpiece is considered. To detect whether their bounding boxes intersect or not and if they do, then there is a collision and the path needs to be re-planned to avoid the collision. While the box of each triangle facet of the workpiece is established to find where the collision takes place. For the detection of light beam occlusion, the box of the incident beam and the reflected beam of the scanner are established (Fig.7.), especially the beam bounding box is equal to the laser beam width. In addition to the triangle facet scanned by the current measuring point, it may miss data if the bounding box of the measuring light and the other triangle facet bounding boxes of the workpiece intersect.

So far the smooth collision-free scanning path we have obtained is on the part frame, the coordinates $\left(X_{p}, Y_{p}, Z_{p}, N_{i}, N_{j}, N_{k}\right)$ must be transformed into $\left(X_{m}, Y_{m}, Z_{m}, B, C\right)$ in the machine frame. The first step is to set up the machine frame, the workpiece frame and the sensor frame (Fig.8.).

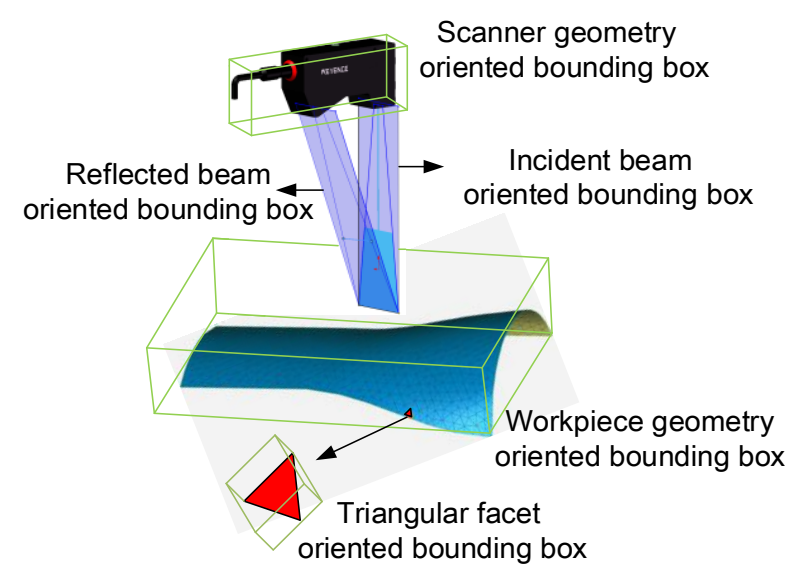

Fig. 7: The establishment of oriented bounding boxes.

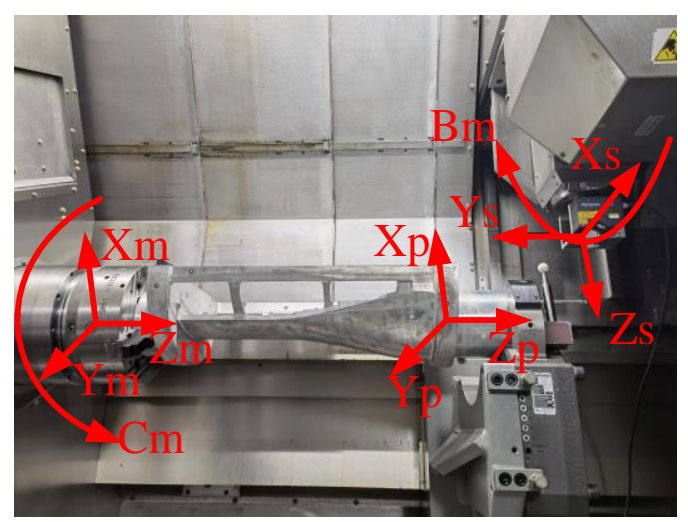

Fig. 8: Definition of the different coordinate system.

In this case, the transformation from sensor frame to machine frame is obtained by calibrating and fitting the standard spherical center. The translation and deflection of the sensor frame relative to the machine frame are calculated by fitting 27 sets of standard spherical center with 3 groups of standard spherical cross-section lines measured under 3 groups of working distance and 3 groups of orientation angle. Therefore, we obtained the translation and deflection from sensor frame to machine frame is $T c$ and $\boldsymbol{D} \boldsymbol{c}$ respectively.

$\boldsymbol{T}_{c}=\left[\mathrm{X}_{\mathrm{T}}, \mathrm{Y}_{\mathrm{T}}, \mathrm{Z}_{\mathrm{T}}\right]^{\mathrm{T}}$

$\boldsymbol{D}_{\boldsymbol{c}}=\left[\mathrm{I}_{\mathrm{T}}, \mathrm{J}_{\mathrm{T}}, \mathrm{K}_{\mathrm{T}}\right]^{\mathrm{T}}$

According to the working distance $\mathrm{H}$, we define that the calibration matrix $\boldsymbol{C}$ is expressed as:

$\boldsymbol{C}=\left[\mathrm{X}_{\mathrm{T}}+\mathrm{H} . \mathrm{I}_{\mathrm{T}}, \mathrm{Y}_{\mathrm{T}}+\mathrm{H} . \mathrm{J}_{\mathrm{T}}, \mathrm{Z}_{\mathrm{T}}+\mathrm{H} . \mathrm{Z}_{\mathrm{T}}\right]$

By measuring the swing angle with the $B$ axis rotation sensor and controlling the rotation of the workpiece with the $\mathrm{C}$ axis, the points can be measured along the normal direction on the 5-axis machine tool. The coordinates of the planned points of a given surface $\left(X_{p}, Y_{p}, Z_{p}, N_{l}, N_{J}, N_{k}\right)$. Since the $C$ axis controls the rotation of the workpiece, the first step is to calculate the angle $R_{c}$ which is the rotation of $\mathrm{c}$ axis corresponding to the normal of the point $\mathrm{Pi}$. The angle $\mathrm{R}_{\mathrm{c}}$ can be calculated as follows:

$R_{\mathrm{c}}=\frac{\arctan \left(\frac{N J}{N I}\right) \times 180^{\circ}}{\pi}$

When the $C$ axis rotates the angle $R_{c}$, the light beam axis of the sensor coincides with the normal direction of the surface point $\mathrm{P}_{i}$ on the XOY plane. Similarly, When B axis rotates the angle $R_{b}$, the light beam axis of the sensor coincides with the normal direction of the surface point $P_{i}$ on the XOZ plane. The difference lies in that the position at $0^{\circ}$ of the $B$ axis is in the opposite direction of the $x$ axis. The angle $R_{b}$ can be expressed as:

$R_{b}=\frac{\arctan \left(\frac{N I}{N K}\right) \times 180^{\circ}}{\pi}+90^{\circ}$

It is worth noting that $\mathrm{B}$-axis controls the spindle swing angle rather than workpiece swing angle. On the contrary, the workpiece is rotated with the rotation of $C$ axis. The rotation matrix $\boldsymbol{R} \boldsymbol{M C}$ and $\boldsymbol{R} \boldsymbol{M B}$ can be expressed as:

$$
\begin{aligned}
R M C & =\left[\begin{array}{ccc}
\cos R_{\mathrm{c}} & -\sin R_{\mathrm{c}} & 0 \\
\sin R_{\mathrm{c}} & \cos R_{\mathrm{c}} & 0 \\
0 & 0 & 1
\end{array}\right] \\
R M B & =\left[\begin{array}{ccc}
\cos R_{b} & 0 & \sin R_{b} \\
0 & 1 & 0 \\
-\sin R_{b} & 0 & \cos R_{b}
\end{array}\right]
\end{aligned}
$$

Eventually, the coordinate transformation can be expressed as:

$\left[\mathrm{X}_{\mathrm{m}}, \mathrm{Y}_{\mathrm{m}}, \mathrm{Z}_{\mathrm{m}}\right]=\left[\mathrm{X}_{\mathrm{p}}, \mathrm{Y}_{\mathrm{p}}, \mathrm{Z}_{\mathrm{p}}\right] \cdot \boldsymbol{R} \boldsymbol{M} \boldsymbol{C}-\boldsymbol{C} \cdot \boldsymbol{R} \boldsymbol{M} \boldsymbol{B}^{\top}$

Then the coordinate of 5 -axis machine tool is obtained:

$\left[X_{m}, Y_{m}, Z_{m}, B, C\right]=\left[X_{m}, Y_{m}, Z_{m}, R_{b}, R_{c}\right]$

\section{APPLICATION}

In order to show the contribution of the proposed path planning method, we propose to develop a scanning application on a 5-axis machine tool to compare the results of 5-axis scanning with the results of 3-axis scanning.

\subsection{Equipment parameters and workpiece information}

Obviously, in the actual application of on-machine measurement, the accuracy of machine tool motion does directly affect the measurement accuracy. In the context of this paper, the measurements with a contact probe and with laser scanner are both obtained using the WFL M35 
machine tool, which is a 5-axis turning-milling composite machining center. According to the detection by laser interferometer, the repeat positioning accuracy of its linear feed axes is about $2-3 \mu \mathrm{m}$, and of the rotary axes is $0.001^{\circ}$. Therefore, the affection of motion accuracy of the processing machine to the measurement accuracy is not taken into account. There is no doubt that the motion accuracy of both the 3 -axis and 5-axis can meet the measurement accuracy requirements, and it has an ignorable impact on the comparison results. The travel of 5axis machine tool spindle is as follows:

Tab. 2: travel of 5-axis machine tool spindle.

\begin{tabular}{ll}
\hline axis & travel \\
\hline $\mathrm{X}$ & $600 \mathrm{~mm}(-20 \ldots+580)$ \\
$\mathrm{Y}$ & $250 \mathrm{~mm}(-100 \ldots+150)$ \\
$\mathrm{Z}$ & $2000 \mathrm{~mm}(0 \ldots+2000)$ \\
$\mathrm{B}$ & $220^{\circ}\left(-110^{\circ} \ldots+110^{\circ}\right)$
\end{tabular}

The OMM system used consists of the KEYENCE LJV7060 sensor mounted in the spindle of the machine tool with a dedicated fixture and tool holder attachment (Fig.8). The value of working distance $\mathrm{H}$ ranges from $52 \mathrm{~mm}$ to 68 $\mathrm{mm}$. Corresponding to FOV, giving the length of laser line $\mathrm{L}=16 \mathrm{~mm}$. The repetition accuracy of the sensor is $0.4 \mu \mathrm{m}$ in the beam axis direction with $5 \mu \mathrm{m}$ in the line laser direction. Besides, the laser scanner uses a $16 \mathrm{~mm}$-wide semiconductor laser probe and it can capture 800 points for each scan, which is to collect a point every $0.02 \mathrm{~mm}$.

In order to meet the application of line laser scanning and show the superiority of path planning, large scale complex surface is chosen to verify the path planning. The surface of this part is included in a $135 \times 380 \times 650 \mathrm{~mm}^{3}$ bounding box, while the maximum normal deflection angle of the surface is $60^{\circ}$. This workpiece is milled by spherical tools with different radius through rough machining, semifinishing machining, and finishing machining. By comparing the result of contact probe measurement with the theoretical CAD model, its machining accuracy is within $0.05 \mathrm{~mm}$ (Fig.9).
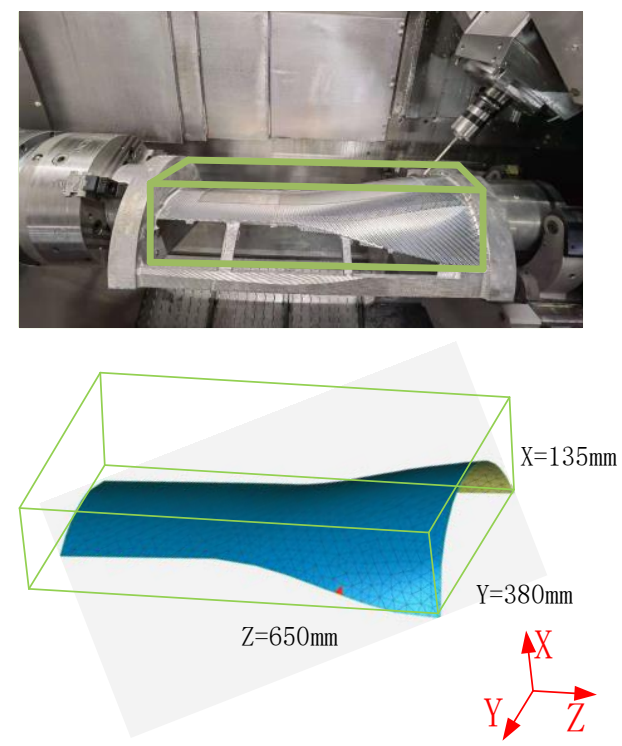

Fig. 9: Probe measurement and workpiece geometry.

\subsection{Experiments}

The scanning efficiency decreases obviously when the overlapping rate of scanning is large or the angle threshold of adjusting position angle is small. At the same time, in order to ensure that there is no lack of vector surface information in the scanning process, we choose the scanning overlap rate is $15 \%$ to ensure the scanning complete surface. The sensor parameters are selected as sampling frequency set to $50 \mathrm{HZ}$ with feed rate set to 120 $\mathrm{mm} / \mathrm{min}$. The orientation angle threshold is adjusted to $10^{\circ}$ to ensure the high quality of surface data in short measurement time.

During the 3-axis scanning process, the beam axis is vertically downward along the $X$ axis, and the machine tool spindle drives the sensor to move in the $Y$ and $Z$ directions. Only when the measurement distance needs to be adjusted to ensure that the curved surface point is within the FOV, the machine tool spindle will be raised or lowered along the $X$ axis. (Fig. 10. (a)). During the 5-axis scanning process, the rotation of the $\mathrm{C}$ axis is increased so that the workpiece can be adjusted, while the rotation of the $B$ axis can adjust the angle of the laser plane of the sensor. ( Fig. 10(b)(c)(d)).

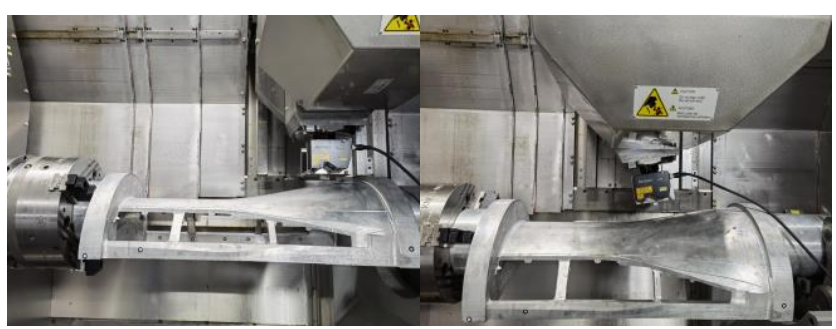

(a) 3 axis scan

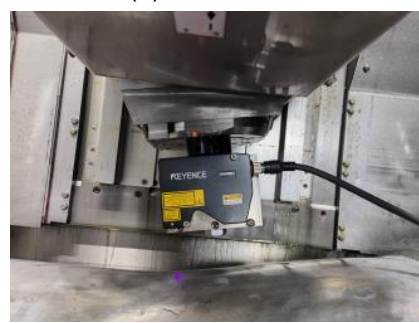

(c) The rotation of the $\mathrm{C}$ axis (b) 5 axis scan

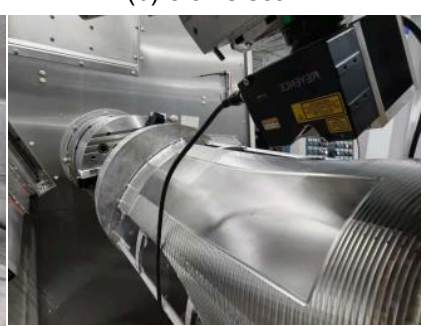

(d) The rotation of the $\mathrm{C}$ axis
Fig. 10: On-machine measurement using line laser scanner

\subsection{Results and discussion}

Using the parameters previously selected. There are 2268 path points in the original 5-axis scan path of the surface, and 36 points are retained after being filtered by the region growing method (Fig. 11.). This also means that the sensor completes 5-axis measurement actions in 36 postures, and each posture measures the corresponding area in an optimized manner without the FOV problem. Moreover, the working distance is out of range in the 3 -axis measurement, which means that the measuring point of the surface falls outside the FOV. Hence further adjustment in the $X$ direction is required. Due to the limitation of the travel of the machine tool, the spindle in the $Y$ direction cannot reach the measuring position so that the whole surface information is not completed. Hence there is part of the surface information as a result of the evaluation. Therefore, a part of the surface is taken to evaluate the precision of the contrast measurement.

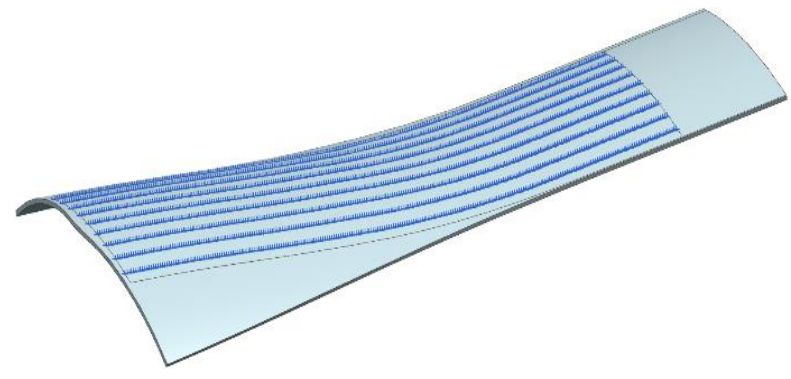


(a) Original scan path of surface in a zig-zag fashion.

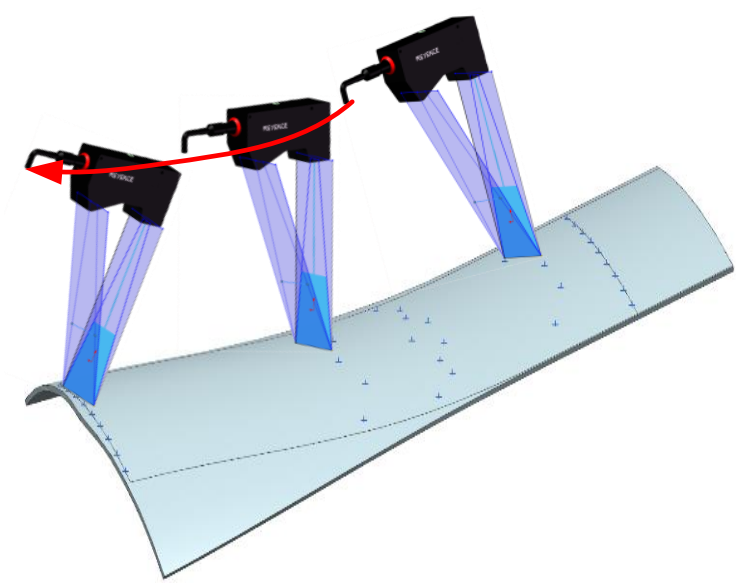

(b) 5-axis scanning path points of surface after filtered.

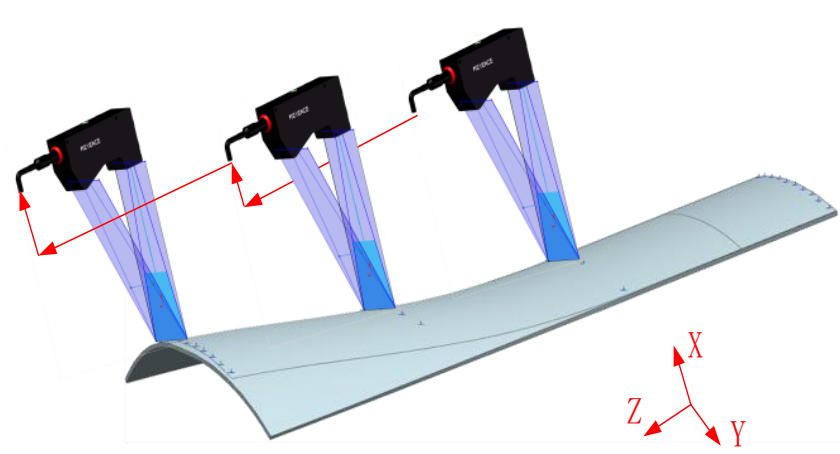

(c) 3-axis scanning path points of surface.

Fig. 11: Surface measured with the two methods.

The result by 3 axis scanning gives a set of 19575242 points while the 5 -axis scanning result has 13960800 points. Both of the point clouds are compared to the part CAD model in order to identify geometrical deviations. Actually, the CAD model in this paper is obtained by surface fitting on the point cloud measurement results of the contact probe. Due to the high accuracy of the probe on-machine measurement and the surface fitting accuracy in the range of $0.02 \mathrm{~mm}$, we believe that this CAD model can represent the actual surface and serve as a comparison object for laser measurement.

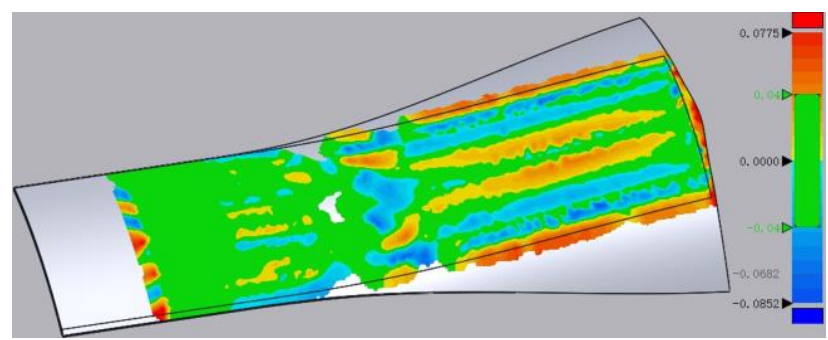

Fig. 12: Geometrical deviation( $\mathrm{mm}$ ) of 3-axis scan.

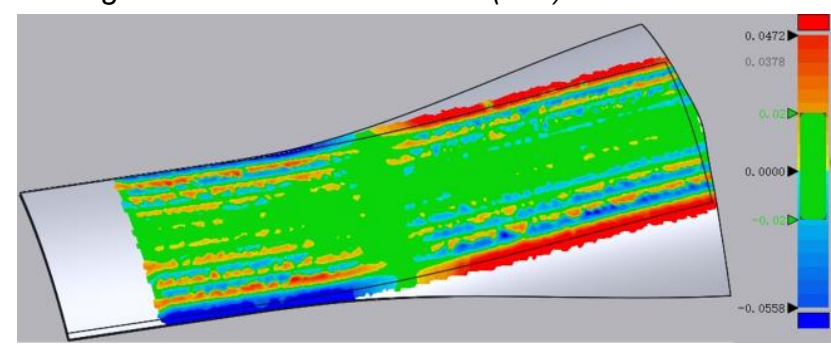

Fig. 13: Geometrical deviation( $\mathrm{mm}$ ) of 5-axis scan.
Since both the sensor orientation and the working distance at each driven point cannot be controlled with the 3 -axis scanning path, the angle between the light beam and the normal vector at each point cannot be limited to the angle threshold range, which is Inferior to the 5-axis scanning path. Hence the scan path length of 5-axis measurement is $3332.9 \mathrm{~mm}$ and that of 3-axis measurement is $4242.2 \mathrm{~mm}$, which means that the measurement efficiency of 5 -axis scanning is improved by $21.4 \%$ in the same feed rate. As shown in Fig. 12 and Fig. 13, the geometric deviation in the overlap region is higher than in the rest of the surface. The geometric deviation of the 3 -axis scan is $0.0494 \mathrm{~mm}$ and that of the 5 -axis scan is $0.0371 \mathrm{~mm}$, which means that the measurement accuracy of 5 -axis scanning is improved by $24.9 \%$. This shows that for the line laser scanning of curved surfaces, the strategy of the 5-axis scanning path not only improves the measuring range and efficiency but also has a positive impact on the measurement accuracy.

\section{CONCLUSION}

To improve the accuracy of line laser on-machine measurement, this paper proposes a path planning method based on the concept of visibility from the CAD model of the inspected part to find correct sensor guidance, which controls the scanning overlap, visual angle, and working distance. The method considers how to set the field of view of the scanner in order to achieve an optimal scanning path. Besides, the angle of rotation and translation value required for the coordinate transformation are calculated from scan paths information. With these values, the curved surface part can automatically be positioned and scanned precisely in a short time using a 5-axis machine tool. By analyzing the comparison experiment results between 3-axis measurement and 5-axis measurement, it can be assessed that the benefits of the proposed path planning method. Compared with the results of contact probe measurement, the measurement deviation is $0.0494 \mathrm{~mm}$ in the 3-axis scan and is reduced to $0.0371 \mathrm{~mm}$ in 5 -axis scan, which shows the enhancement of the efficiency by $21.4 \%$ and the accuracy by $24.9 \%$.

The proposed method is more applicable to inspection because the actual CAD model is generated from reverse engineering. Future work will be dedicated to the application and evaluation of adapted scan paths other than zig-zag with unknown parts.

\section{REFERENCES}

[Liu 2014] Liu. Y.Q., et al. Accurate measurement and digital modeling of complex surface. Applied Mechanics and Materials, 2014, Vol.3558. ISBN 16609336

[John 2007] John S.A, Du, H. Assuring the Day-to-Day accuracy of coordinate measuring machines-a comparison of tools and procedures. Journal of Manufacturing Processes, 2007, Vol.9, No.2. ISBN 15266125

[Thomas 2018] Thomas O.H., et al. A non-contact laser speckle sensor for the measurement of robotic tool speed. Robotics and Computer Integrated Manufacturing,2018, Vol.53, pp 187-196. ISBN 07365845

[Christian 2018] Christian M., et al. Accurate light source position estimation for a laser triangulation measurement device using particle swarm optimization. Measurement, 2018, Vol.125, pp 406-414. ISBN 02632241

[Nooraniazad 2011] Nooraniazad M., et al. Accurate light source position estimation for a laser triangulation measurement device using particle swarm optimization. AIP Conference Proceedings, 2011, pp 1570 
[Mahmud 2010] Mahmud M., et al. 3D part inspection path planning of a laser scanner with control on the uncertainty Computer-Aided Design,2010, Vol.43, No.4. pp 345-355. ISBN 00104485

[Isheil 2011] Isheil A., et al. Systematic error correction of a 3D laser scanning measurement device. Optics and Lasers in Engineering,2011; Vol.49, No.1.pp 16-24.

[Son 2002] Son S., et al. Automated laser scanning system for reverse engineering and Inspection. International Journal of Machine Tools and Manufacture, 2002, Vol.42, No.8. ISBN 08906955

[Xi 1999] Xi F and Shu C. Cad-based path planning for 3-d line laser scanning. Computer-Aided Design, 1999, Vol.31, No.7. ISBN 00104485

[Wu 2015] Wu Q., et al. Path planning for surface inspection on a robot-based scanning system. IEEE International Conference on Mechatronics and Automation (ICMA),2015, pp 2284-2289
[Xi 2001] Xi F., et al. Error compensation for threedimensional line laser scanning data. International Journal of Advanced Manufacturing Technology. 2001; Vol.43 18. pp 211-216.

[Phan 2018] Phan, N. D. M., et al. Scanner path planning with the control of overlap for part inspection with an industrial robot. The International Journal of Advanced Manufacturing Technology, 2018, Vol.98, No.1-4, pp 629643. ISBN 02683768

[Ahmet 2010] Ahmet C., et al. A novel iso-scallop tool-path generation for efficient 5 -axis machining of free-form surfaces. The International Journal of Advanced Manufacturing Technology, 2010, Vol.51, No.9-12, pp 1083-1098. ISBN 02683768

[Li 2011] Li W., et al. Tool path generation for triangular meshes using least-squares conformal map. International journal of production research, 2011, Vol.49, No. 12. ISBN 00207543 